\title{
Highlights on Diabetes Mellitus in Older Adults
}

\author{
Shereen Moustafa Mousa, MD' \\ Geriatrics \& Gerontology Department, Faculty of Medicine, Ain Shams University
}

\section{Background}

It is estimated that by the year 2030, there will be 8.6 million adults with diabetes in Egypt, making it the country with the tenth largest population of diabetics in the world [1]. The prevalence of diabetes rises dramatically with age in both sexes; it reaches almost 20\% among females aged 50-59 [2]. The burden of diabetes in adults is often described in terms of its impact on working-age, while in older adults it is linked to decreased functional status, institutionalization, and mortality [3]. Older persons and/or those with multiple comorbidities have often been excluded from randomized controlled trials of treatment despite having the highest prevalence of diabetes [4].

\section{Can diabetes mellitus be prevented?}

Diabetes mellitus could be prevented in older adults. The American Diabetes Association (ADA) [5] recommends testing to detect type 2 diabetes and prediabetes in the following conditions:

- Asymptomatic people who are overweight or obese (BMI $\geq 25 \mathrm{~kg} / \mathrm{m} 2$ ) and who have one or more additional risk factors for diabetes (physical inactivity, first-degree relative with diabetes).

- High-risk race/ethnicity.

- Hypertension.

- HDL cholesterol level $<35 \mathrm{mg} /$ and/or a triglyceride level $>250 \mathrm{mg} / \mathrm{Dl}$.

- Other clinical conditions associated with insulin resistance (e.g., severe obesity, acanthosis nigricans).

- History of cardiovascular disease (CVD).

In those without these risk factors, testing should begin at age 45 years. If tests are normal, repeating testing at least at 3-year intervals is reasonable. To test for diabetes or prediabetes, the Hemoglobin A1C (A1C) (5.7-6.4\%),
Fasting Blood Sugar (FPG) $100 \mathrm{mg} / \mathrm{dL}$ to $125 \mathrm{mg} / \mathrm{dL}$, or $75-\mathrm{g} 2-\mathrm{h}$ (Oral Glucose Tolerance test) OGTT 140 $\mathrm{mg} / \mathrm{dL}$ to $199 \mathrm{mg} / \mathrm{dL}$ are appropriate. In the Diabetes Prevention Program (DPP), which is the largest trial to date, $20 \%$ of participants were aged 60 years at enrollment. These participants seemed to have more efficacies from the lifestyle intervention than younger participants, but did not appear to benefit from metformin [6].

Diagnosis of diabetes mellitus in elderly [7]:

- $\mathrm{A} 1 \mathrm{C} \geq 6.5 \%$. The test should be performed in a laboratory using a method that is certified and standardized to the Diabetes Control and Complications Trial (DCCT) assay; or

- $\quad F P G \geq 126 \mathrm{mg} / \mathrm{dL}$. Fasting is defined as no caloric intake for at least $8 \mathrm{~h}$; or

- 2-h plasma glucose $\geq 200 \mathrm{mg} / \mathrm{dL}$ during an OGTT). The test should be performed as described by the WHO, using a glucose load containing the equivalent of $75 \mathrm{~g}$ anhydrous glucose dissolved in water; or 
- In a patient with classic symptoms of hyperglycemia or hyperglycemic crisis, a random plasma glucose $\geq 200 \mathrm{mg} / \mathrm{dL}$

- In the absence of unequivocal hyperglycemia, result should be confirmed by repeat testing.

Treatment Goals for Glycemia, Blood Pressure, and Dyslipidemia in Older Adults with Diabetes:

The consensus report of the American Geriatric Society [4] regarding diabetes in older adults sets treatment goals according to the patient's characteristics and health status.

For healthy elderly (Few coexisting chronic illnesses, intact cognitive and functional status):

Reasonable A1C Goal is $<7.5 \%$ (A lower goal may be set for an individual if achievable without recurrent or severe hypoglycemia or undue treatment burden), FPG or preprandial (90$130 \mathrm{mg} / \mathrm{dl})$, Bedtime glucose (90$150 \mathrm{mg} / \mathrm{dl}$ ), Blood Pressure < 140/80, Lipids (statins unless contraindicated or not tolerated).

- For Complex/intermediate elderly (Multiple coexisting chronic illnesses or 2+ instrumental ADL impairments or mild to moderate cognitive impairment):

A1C $<8.0 \%$, FPG or pre-prandial (90$150 \mathrm{mg} / \mathrm{dl})$, Bedtime glucose (100$180 \mathrm{mg} / \mathrm{dl}$ ), Blood Pressure < 140/80, Lipids (statins unless contraindicated or not tolerated).

- Very complex/poor health elderly (Long-term care or end-stage chronic illnesses or moderate to severe cognitive impairment or 2+ ADL dependencies):

A1C $<8.5 \%$, FPG or preprandial (100$180 \mathrm{mg} / \mathrm{dl})$, Bedtime glucose (110200mg/dl), Blood Pressure < 150/90,
Lipids (Consider likelihood of benefit with statin(secondary prevention more than primary)).

The U.S. Department of Veterans Affairs and the U.S. Department of Defense (VA/DOD)

Recommend glycemic goals based on comorbidity and life expectancy as follows [8]:

- Patient with either none or very mild microvascular complications, free of major concurrent illnesses and has a life expectancy of at least 10-15 years, should have an A1C target of $<7 \%$,if it can be achieved without risk.

- Patient with longer duration of diabetes (more than 10 years) or with comorbid conditions and who requires a combination medication regimen including insulin should have an A1C target of $<8 \%$.

- Patient with advanced microvascular complications and/or major comorbid illness and/or a life expectancy of less than 5 years is unlikely to benefit from aggressive glycemic control and should have an A1C target of $8-9 \%$. Lower targets $(<8 \%)$ can be established on an individual basis.

Hypoglycemic drug use in elderly:

- It should be individualized yet Metformin is often considered the firstline therapy in type 2 diabetes due to its low risk for hypoglycemia. The dose should be reduced if estimated glomerular filtration rate (eGFR) is 30 $60 \mathrm{~mL} / \mathrm{min}$, and the drug should not be used if eGFR is $<30 \mathrm{~mL} / \mathrm{min}$ [9].

- Sulfonylureas carry the risk of hypoglycemia which is problematic for older patients. Glyburide has the highest hypoglycemia risk and should not be prescribed for older adults [10].

- Glinides, a-Glucosidase inhibitors, Dipeptidyl peptidase-4 inhibitors, Glucagon-like peptide-1 agonists target 
more the postprandial hyperglycemia [4].

- Insulin therapy can be used in selected older adults with type 2 diabetes with similar efficacy and hypoglycemia risk as in younger patients. However, the risk of hypoglycemia must be considered before using an insulin regimen to achieve an aggressive target for hyperglycemia control [4].

Elderly owe independent risk factors for hypoglycemia which include hospital discharge within the prior 30 days, advanced age, black race, and use of five or more concomitant medications [11]. Finally every diabetic elderly should have consultation regarding nutrition and physical activity and should be screened for chronic diabetes complications, cognitive impairment, functional impairment, falls, polypharmacy, depression, visual and hearing impairment [4].

\section{References:}

1- Shaw JE, Sicree RA, Zimmet PZ. Global estimates of the prevalence of diabetes for 2010 and 2030. Diabetes Res Clin Pract 2010;87(1):4-14.

2- Arafa NA and Amin GE. The Epidemiology of Diabetes Mellitus in Egypt: Results of a National Survey. The Egyptian Journal of Community Medicine 2010; 28 (3):29-43.

3- Brown AF, Mangione CM, Saliba D et al. California Healthcare Foundation /American Geriatrics Society Panel on Improving Care for Elders with Diabetes. Guidelines for improving the care of the older person with diabetes mellitus. J Am Geriatr Soc 2003;51: S265-S280.

4- Kirkman MS, Briscoe VJ, Clark $\mathrm{N}$ et al. Diabetes in Older Adults: A Consensus Report. Special article by the American Diabetes Association and the American Geriatrics Society.JAGS 2012

5- AMERICAN DIABETES ASSOCIATION. Standards of Medical Care in Diabetesd2013 position statement.

DIABETES CARE;2013: 36 (1): S11-S66.

6- Crandall J, Schade D, Ma Y et al. Diabetes Prevention Program Research Group. The influence of age on the effects of lifestyle modification and metformin in prevention of diabetes. J Gerontol A Biol Sci Med Sci 2006;61A:1075-1081.

7- Executive Summary: Standards of Medical Care inDiabetesd2013. DIABETES CARE;2013: 36 (1): S4-S10.

8- United States Department of Veterans Affairs. VA/DOD clinical practice guidelines: Management of diabetes mellitus in primary care, 2010.

9- Lipska KJ, Bailey CJ, Inzucchi SE. Use of metformin in the setting of mild-to-moderate renal insufficiency. Diabetes Care 2011;34:1431-1437.

10- American Geriatrics Society. Beers Criteria Update Expert Panel. American Geriatrics Society updated Beers Criteria for potentially inappropriate medication use in older adults. J Am Geriatr Soc 2012(60):616-631.

11- Shorr RI, Ray WA, Daugherty JR et al. Incidence and risk factors for serious hypoglycemia in older persons using insulin or sulfonylureas. Arch Intern Med 1997; 157:1681-1686. 